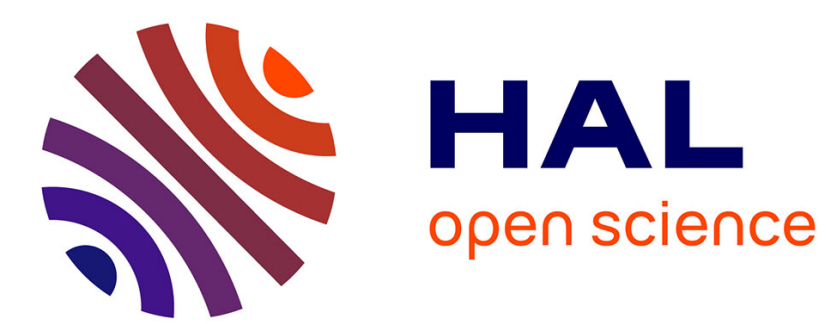

\title{
À la table des élites bordelaises du XVIIIe siècle
}

Philippe Meyzie

\section{To cite this version:}

Philippe Meyzie. À la table des élites bordelaises du XVIIIe siècle. Annales du Midi: revue archéologique, historique et philologique de la France méridionale, 2003, 115 (241), pp.69-88. 10.3406/anami.2003.2799 . hal-02264312

\section{HAL Id: hal-02264312 \\ https://hal.science/hal-02264312}

Submitted on 6 Aug 2019

HAL is a multi-disciplinary open access archive for the deposit and dissemination of scientific research documents, whether they are published or not. The documents may come from teaching and research institutions in France or abroad, or from public or private research centers.
L'archive ouverte pluridisciplinaire HAL, est destinée au dépôt et à la diffusion de documents scientifiques de niveau recherche, publiés ou non, émanant des établissements d'enseignement et de recherche français ou étrangers, des laboratoires publics ou privés. 


\section{À la table des élites bordelaises du XVIIIle siècle}

Philippe Meyzie

\section{Citer ce document / Cite this document :}

Meyzie Philippe. À la table des élites bordelaises du XVIIle siècle. In: Annales du Midi : revue archéologique, historique et philologique de la France méridionale, Tome 115, N²41, 2003. La culture matérielle dans le Midi de la France à l'époque moderne. pp. 69-88;

doi : https://doi.org/10.3406/anami.2003.2799

https://www.persee.fr/doc/anami_0003-4398_2003_num_115_241_2799

Fichier pdf généré le 18/04/2018 


\begin{abstract}
At the Table of Bordeaux's Elites in the Eighteenth Century.

The eighteenth century, veritable golden age for Bordeaux, is a good time for studying the originality of food tastes and table manners of social elites. Close to the sea, open to foreign cultural influences, with a vast hinterland offering gastronomic riches, Bordeaux had much culinary potential. Through refinement, luxury and the search for good taste, food customs appear as a social stake and as a criterion of distinction between nobility and merchant groups. By associating tradition and modernity, the tables of Bordeaux's social elites thus contributed to the emergence of a specific culinary identity.
\end{abstract}

\title{
Résumé
}

Le XVIIle siècle, véritable âge d'or pour Bordeaux, est un moment privilégié pour étudier l'originalité des goûts alimentaires et des manières de table des élites sociales. La proximité de la mer, l'ouverture aux influences culturelles étrangères et les richesses gastronomiques d'un vaste arrière-pays offrent de nombreuses potentialités culinaires. À travers luxe, raffinement et recherche du bon goût, les habitudes alimentaires apparaissent comme un enjeu social et un critère de distinction entre noblesse et négoce. En associant tradition et modernité, la table des élites bordelaises contribue ainsi à l'émergence d'une identité culinaire spécifique. sociales. La proximité de la mer, l'ouverture aux influences culturelles étrangères et les richesses gastronomiques d'un vaste arrière-pays offrent de nombreuses potentialités culinaires. À travers luxe, raffinement et recherche du bon goût, les habitudes alimentaires apparaissent comme un enjeu social et un critère de distinction entre noblesse et négoce. En associant tradition et modernité, la table des élites bordelaises contribue ainsi à l'émergence d'une identité culinaire spécifique.

\section{Zusammenfassung}

Zu Tisch bei den Eliten von Bordeaux im 18. Jahrhundert.

Das 18. Jahrhundert war für Bordeaux ein wahrhaft Goldenes Zeitalter und bietet sich dazu an, die besonderen Gaumenfreuden und die Tischsitten der gesellschaftlichen Eliten zu studieren. Die Nähe zum Meer, die Offenheit gegenüber kulturellen Einflüssen aus dem Ausland sowie der gastronomische Reichtum eines weiträumigen Hinterlandes eröffneten zahlreiche kulinarische Möglichkeiten. Die Ernährungsgewohnheiten erscheinen unter Berücksichtigung des Luxus und der Suche nach dem Gaumenkitzel als ein gesellschaftliches Phänomen und als ein Unterscheidungskriterium zwischen dem Adel und der Kaufmannschaft. Die Tafel der Eliten verband Tradition und Modernität und trug so zur Ausformung eines besonderen kulinarischen Identität bei.

\section{Resumen}

Cómo comen las elites bordelesas del siglo XVIII.

El siglo XVIII, verdadera edad de oro para Burdeos, es un momento privilegiado para estudiar la originalidad de los gustos culinarios y las buenas maneras en el comer entre las elites sociales. La cercanía del mar, la apertura de la ciudad a las influencias culturales extranjeras y las riquezas gastronómicas de la región entrañan grandes potencialidades culinarias. Mediante el lujo, el refinamiento y la búsqueda del buen gusto, las costumbres culinarias vienen a ser un alarde de rango social y un criterio para distinguir entre nobleza y negocio. Al asociar tradición y modernidad, la mesa de las elites bordelesas contribuye a la aparición de una identidad culinaria propia.

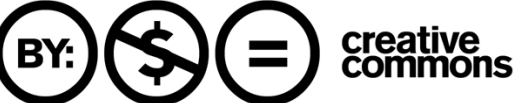




\section{Philippe MEYZIE*}

\section{À LA TABLE DES ÉLITES BORDELAISES DU XVIII' SIEECLE}

«Les Bordelais semblent être grands amateurs de théâtre ; mais, d'après ce que $\mathrm{j}$ 'ai appris, leur distraction et leur luxe consistent surtout dans la bonne chère et les dîners'. »C'est ainsi qu'en 1804 Adriaan Van der Willigen, voyageur hollandais de passage, vante les plaisirs de la table bordelaise. Cette évocation de la cuisine est un trait récurrent des récits de voyageurs séjournant dans la ville. Or, le jugement de Van der Willigen concerne le début du $\mathrm{XIX}^{\mathrm{c}}$ siècle, fin de l'âge d'or bordelais ${ }^{2}$. Il est donc intéressant de porter notre regard sur la table des élites bordelaises à son apogée, c'est-à-dire entre les années 1720 et 1770 . Sous le terme d'élites, nous n'entendons pas uniquement les plus riches personnages de la ville, mais tous ceux qui possèdent un prestige social et une notabilité importante. Négociants et nobles, même désargentés, seront donc au cœur de notre étude sur les goûts alimentaires et les manières de table.

Cette approche du thème de l'alimentation sous l'angle des choix culinaires permet, comme le souligne Jean-Louis Flandrin, de « chercher quels rapports les goûts d'un peuple, d'une époque, d'un groupe ont entretenus avec les autres caractéristiques de sa culture et avec ses fondements matériels ${ }^{3}{ }^{\prime}$. Dans le Bordeaux du XVIII ${ }^{e}$ siècle, quelles sont donc les caractéristiques essentielles et

* 3, allée des Foreurs, 33400 Talence.

1. AmTMann (Th.), "Les impressions d'un Hollandais à Bordeaux en 1804 ", Revue historique de Bordeaux, 1913, p. 263.

2. Cocul.A (Anne-Marie). «Ce siècle avait deux ans, ou Bordeaux d'un siècle à l'autre », dans Merlio (G.) et Peilietier (N.) dir., Bordeaux au temps de Hölderlin, Paris, 1997, p. 9.

3. Flandrin (Jean-Louis), Chronique de Platine : pour une gastronomie historique, Paris, 1992. p. 319.

Agrégé d'histoire, allocataire-moniteur à l'Université Michel-de-MontaigneBordeaux-III, Philippe Meyzie prépare une thèse sur " les goûts alimentaires, les manières de table et la société dans le Sud-Ouest aquitain (XVIII'-milieu du XIX ${ }^{\mathrm{c}}$ siècle) $»$. 
l'originalité éventuelle de la table des élites, et comment sa fonction sociale participe-t-elle à l'évolution et à l'affirmation de son identité ?

Le dynamisme des élites urbaines et l'attrait exercé par Bordeaux au XVIII siècle nous offrent de nombreuses sources sur les activités culinaires. Récits de voyage, inventaires après décès, livres de comptes ou menus permettent, de façon plus ou moins directe, de saisir cette réalité quotidienne souvent inaccessible. Grâce à ces sources, l'analyse locale peut s'avérer fructueuse.

Sans vouloir s'inscrire dans une perspective déterministe, il conviendra tout de même de souligner, dans un premier temps, comme le fait Van der Willigen en évoquant «un pays de cocagne" », que nombreux sont les atouts favorables au développement des plaisirs culinaires chez les élites bordelaises. Le luxe et le raffinement de la table, signes importants de distinction sociale, façonnent aussi l'identité de la cuisine locale. Nous verrons enfin que cette table, enjeu des rapports sociaux, connaît un évolution particulière qui oscille entre modernité culinaire et tradition des saveurs.

\section{Une capitale gastronomique provinciale au XVIII" siècle}

Le XVIII" siècle est un "âge d'or » pour Bordeaux" comme l'attestent sa richesse économique et son dynamisme démographique. Cet essor participe pleinement au développement d'une gastronomie locale mise en valeur par les élites. Proximité de la mer, présence de la Garonne, vaste arrière-pays, diversité culturelle permettent la mise en place de goûts alimentaires et de manières de table originaux.

\section{Une table de qualité}

Les nombreux dîners mentionnés dans nos sources et l'attention des voyageurs étrangers aux plaisirs de la table sont autant de témoignages sur le goût des élites bordelaises pour la gastronomie.

À cause de son dynamisme économique, le port de Bordeaux est une destination privilégiée pour les voyageurs étrangers, qui s'installent le plus souvent dans des familles de la ville. Ainsi Madame de La Roche, lors de son passage en 1785, séjourne dans l'hôtel particulier de la famille du négociant Bethmann", dont elle peut évoquer les mérites culinaires : "Cela se passe comme dans les bonnes maisons de chez nous, si ce n'est, je crois, qu'à raison

4. Amtani (Ph.) art. cit.. p. 263.

5. Butlal. (Paul) et Poussou (Jean-Pierre). La vie quotidienne a Bordeand au XVII' sicele, Paris, 1980. P. 9.

6. MrALIDRE de L_APO(YADE. «Impression d'une Allemande à Bordeaux en 178.5 ". Rerue historicute de Bordeant, 19)11, p. 269. 
de la facilité qu'on a d'ajouter du poisson de mer aux ragoûts et aux rôtis, les menus sont plus abondants. Après deux potages, chacun prit et offrit de ce qu'il voulut de chacun des plats qui étaient devant lui. Nous avions cinq espèces des meilleurs poissons de mer, entre autres des rougets, petits poissons rouges à la chair très délicate et qui ressemblent aux dorades de la Chine. Nous avions aussi des tourterelles, une par personne, ainsi qu'on fait chez nous des grives. L'habitude qu'on a de servir le lièvre avec la tête fait que cet animal, mis à côté du turbot, qui est un poisson plat et rond, offre un drôle d'aspect. Les asperges et les petits pois sont les seuls légumes qu'on trouve pendant la sécheresse. Tout était très réussi, en particulier les pâtisseries, qui furent servies avec beaucoup de glaces; les messieurs burent des vins exquis?. " Cette voyageuse allemande, sensible à la cuisine bordelaise, souligne déjà les caractéristiques majeures d'une table marquée par l'abondance, la proximité de l'Océan, les préférences pour le gibier et bien entendu un goût pour des vins de qualité.

Souvent la description des repas est mise en parallèle avec la volonté des élites d'affirmer leur statut social. Thomas Jefferson, en voyage en France dans les années 1780 , n'hésite pas à évoquer « un exhibitionnisme des familles patriciennes ${ }^{x} »$. Cette opinion est reprise quelques années plus tard par un autre voyageur, Miranda, qui voit dans le luxe de la table « tout le faste d'une cour corrompue" ». Au-delà d'une vision commune sur la qualité et la diversité des mets proposés, on voit apparaître un lien important entre la table et la société des élites dans son goût du luxe et de la distinction sociale.

Il n'est donc pas surprenant de retrouver dans nos sources la mention de nombreux repas organisés tant par des particuliers que dans un cadre public. Les Chroniques bordelaises de Lamontaigne notent en nombre ces repas somptueux offerts par les grands personnages de la ville, comme le souper donné par le président Lalande le 13 juillet $1760^{10}$ ou celui, suivi d'un bal, proposé par l'intendance le 8 janvier $1762^{\prime \prime}$. La jurade et la chambre de commerce mettent sur pied aussi régulièrement des banquets. Ces élites bourgeoises ont alors souvent recours à des traiteurs renommés, comme Bardineau, pour apprêter des festins à l'occasion de la venue d'un grand personnage comme l'intendant ou pour la victoire des armées du roi ${ }^{12}$. Ces institutions nous ont aussi laissé des repas plus quotidiens, réservés aux membres de la chambre de commerce, mais là encore préparés par un traiteur. Les factures conservées mentionnent une dizaine de repas durant les années

7. Ibid.

8. Ginisthe (B.). Thomas Jefferson à Bordeaux, Bordeaux, 1996.

9. BAul_iy (O.). « Miranda et Bordeaux », Revue historique de Bordeaux, 1966, p. 125.

10. Lamontaigin: (F. de). Chroniques bordelaises, Bordeaux, 1926, p. 39.

11. Ibid.. p. 91.

12. Archives départementales de la Gironde. C 1044, liste et dates des repas servis à l'hôtel de ville. 
$1745-1754^{13}$. Ces menus nous permettent de percevoir au plus près les goûts alimentaires des élites à travers les plats consommés. La qualité des mets servis par des professionnels de la table peut être considérée comme le reflet de la haute gastronomie bordelaise du XVIII' siècle. Ces traiteurs se rapprochent des activités de la corporation des maîtres hôteliers de Bordeaux, dans laquelle chaque futur maître doit présenter un chef-d'œuvre culinaire. Ils confectionnent, par exemple, des plats d'oreilles de veau à la poivrade, des pigeons aux écrevisses, des tourtes de pigeons garnis de ris de veau, autant de préparations qui suggèrent la créativité gastronomique et les possibilités culinaires offertes par ces métiers de bouche aux palais des élites de la ville.

Les récits de voyage et les repas édilitaires nous ont donc permis de voir que la table tenait une place importante dans la vie des élites du XVIII ${ }^{\circ}$ siècle. Cette cuisine bordelaise raffinée sait très bien tirer profit d'un environnement favorable.

\section{De nombreux atouts gastronomiques}

En effet, l'identité culinaire régionale ne saurait se dégager des conditions qui lui sont proposées par la nature ou par son environnement culturel. Toutefois il ne faut pas surestimer le poids de ces influences extérieures car les élites bordelaises, par leur richesse et leur statut social, demeurent libres de leurs choix alimentaires et donc de la construction de leurs goûts culinaires. La mer et la Garonne, ainsi que la présence de nombreuses colonies étrangères, contribuent évidemment à façonner la table des élites de la ville, même si ces influences ne sont jamais déterminantes.

Ainsi, il peut être intéressant d'évaluer l'impact du milieu naturel sur les choix alimentaires. Pour cela, il nous a semblé judicieux de nous attarder sur le cas du poisson et des produits de la mer en général. En effet, comme le rappelle Michel Figeac, «la proximité de l'Océan est visible dans les formes de consommation, en particulier sur les tables des élites bordelaises ${ }^{14} »$. Toutefois il convient d'être prudent. Dans les inventaires après décès que nous avons dépouillés ${ }^{15}$, les poissonnières n'apparaissent que dans $50 \%$ des cas. Ce pourcentage modeste ne saurait cacher une consommation importante de poisson, mais s'explique par le goût particulier des élites bordelaises pour les poissons en ragoût, en terrine ou accompagnant des viandes, recettes qui ne nécessitent pas un recours systématique à cet ustensile. Dans les menus de la jurade et de la chambre de commerce, nous rencontrons en effet de nombreuses

13. ADG, C 4421 .

14. Figliac (Michel), «Bordeaux porte océane dans les récits de voyage d’A. Young à Stendhal », dans Bordeaux, porte océane, Bordeaux, 1999, p. 442.

15. 16 inventaires pour la période 1720-1725.16 inventaires pour la période 1770-1775. 
terrines de soles ${ }^{16}$, des pâtés chauds d'anguilles ou des soles en ragoût ${ }^{17}$. Madame de La Roche évoque elle aussi la diversité des poissons présentés sur les tables et le goût particulier de ses hôtes pour les préparations de poisson sous forme de ragoût ou mélangé aux viandes ${ }^{18}$. Les plats mariant produits de la mer et viandes sont très prisés. Poulardes aux huîtres ${ }^{19}$, pigeons aux écrevisses $^{20}$ ou filet de bœuf aux anchois ${ }^{21}$ semblent particulièrement aiguiser les papilles bordelaises. Les produits de la Garonne comme les écrevisses ou les anguilles sont sans doute un trait caractéristique de l'alimentation locale. Donc nombreux sont les poissons servis : soles, saumons, carpes se côtoient sur les tables ; reflet des possibilités offertes par l'Océan, la Garonne ou les étangs du Médoc, ils sont aussi la base d'une identité culinaire propre.

L'Océan procure à la table bordelaise de nombreux mets ; il fait aussi de la ville un pôle économique et commercial majeur. Les goûts alimentaires des élites profitent donc des produits fournis par un arrière-pays proche et par des horizons beaucoup plus lointains. La prédilection des élites bordelaises pour certains aliments montre bien que le Sud-Ouest est pour leur table un réservoir important de mets et de victuailles variés. Le goût pour les truffes en est un bon exemple, car ce produit est emblématique de l'arrière-pays agricole de la capitale régionale qu'est Bordeaux. En effet, même si la truffe du Périgord n'a connu qu'un succès tardif en France au XVIII' ${ }^{c}$ siècle ${ }^{22}$, elle apparaît souvent et de manière précoce dans les repas bordelais. Un dîner servi le 21 juillet 1715 à la jurade comprend, entre autres, " une tourte de lapereaux garnie de six truffes ${ }^{2.3} »$. Dans les menus préparés par les traiteurs à la chambre de commerce, les truffes sont présentées nature en même temps que les salades et les desserts ${ }^{24}$. Elles sont aussi souvent utilisées comme garniture des volailles dans des préparations très appréciées comme le chapon aux truffes.

Cependant, dans une ville cosmopolite, les influences étrangères jouent elles aussi un rôle fondamental dans la construction d'une identité culinaire. L'activité commerciale du port et la présence d'importantes colonies étrangères ne sont pas sans donner à la table quelques saveurs particulières. En premier lieu, l'échelle du trafic commercial donne la possibilité de consommer des produits parfois lointains. La cave de l'ingénieur du roi Saint-André25, influencé par les goûts de la ville dans laquelle il séjourna durant plusieurs années, révèle bien à travers les origines variées des vins et des liqueurs,

16. ADG. C 1048, repas du 20 mai 1757.

17. Archives municipales de Bordeaux. AA 25, repas pour l'ouverture du parlement le 12 septembre 1740 .

18. MEAUdRE de LAPOUYADE, art. cit., p. 269.

19. ADG, C 4424, dîner servi le 15 septembre 1775 à la chambre de commerce.

20. ADG, C 1764, livre de délibérations des maîtres hôteliers de Bordeaux, 21 août 1773.

21. AMB, CC 1114, repas du 19 septembre 1747.

22. Aquitaine : produits du terroir et recettes traditionnelles, Paris, 1997, p. 200.

23. AMB, CC 1114, menues dépenses de l'hôtel de ville.

24. ADG, C 4421 .

25. ADG, 3 E 13064. 11 janvier 1775. 
aliments facilement transportables, l'étendue de l'ouverture du goût proposée aux élites. S'y côtoient en grandes quantités des vins du royaume, comme ceux de Bourgogne, de Cognac ou de Perpignan, et des vins provenant de régions plus lointaines, comme le marasquin de Venise, les vins d'Alicante, de Madère ou de Rancio. Si on compare cette cave à celle de Claude-Philippe Fyot de La Marche, premier président du parlement de Dijon, on constate que cette dernière ne comprend que peu de vins étrangers et aucune bouteille de vin de Bordeaux ${ }^{26}$. La diversité des vins consommés par les élites bordelaises est donc bien une spécificité. La présence d'autres boissons, comme l'eau-de-vie ou la bière, souligne quant à elle l'influence culturelle étrangère sur leur table. Les menues dépenses de l'hôtel de ville signalent par exemple l'achat régulier de bière durant les mois d'été et même quelquefois en hiver ${ }^{27}$. Les comptes de la chambre de commerce ${ }^{28}$ confirment cette consommation, qui peut paraître surprenante dans une région réputée pour ses vins. Or ce goût pour la bière est une spécificité bordelaise, puisque cette boisson est peu appréciéc dans les autres villes comme Paris. On peut donc penser que l'influence culturelle des Hollandais, des Allemands et des Anglais, peuples friands de bière, relayée par les circuits du commerce et la présence de colonies étrangères, est à l'origine de ce goût particulier.

Diversité des produits régionaux, influences culinaires étrangères, attrait pour les plaisirs de la table sont autant de facteurs qui permettent le développement de goûts alimentaires et de manières de table originaux chez les élites bordelaises. Cependant, afin que cette gastronomie locale se manifeste pleinement et demeure en évolution permanente, il faut que la table apparaisse comme un enjeu social pour ces élites.

\section{Luxe et raffinement de la table}

Au cœur des pratiques sociales, la table possède, à Bordeaux comme ailleurs, un rôle majeur de distinction sociale ${ }^{29}$. Les élites cherchent à se différencier du reste de la société, mais aussi entre elles, face à l'émergence de nouveaux groupes. Dans le cadre de Bordeaux au XVIII' siècle, cette approche offre un double intérêt : saisir les rapports entre noblesse et négoce au niveau des goûts alimentaires et des manières de table, mais aussi mesurer le rôle social de la table et surtout voir si celui-ci conduit à des pratiques culinaires originales.

26. Ponsot (P.), « Les bouteilles du président : les boissons d'un parlementaire bressan-bourguignon au XVIII" siècle», dans GARRItr (Gilbert) dir., Le vin des historiens. Actes du premier symposium Vin et Histoire. Suze-La-Rousse, 1989, p. 153-160.

27. $\mathrm{AMB}, \mathrm{CC} 1114$.

28. $\triangle \mathrm{DG}, \mathrm{C} 4425$.

29. FI ANDrIX (Jean-Louis), "La distinction par le goût », dans ArIís (Philippe) et DI BY (Georges) dir.. Histoire de la vie privée de la Renaissance aux Lamières, Paris, 1986. p. 267-3099. 


\section{Luxe et abondance}

Comme nous avons pu déjà le constater à travers le regard porté par Miranda sur la table bordelaise ${ }^{30}$, les élites de la ville semblent manifester un goût particulier de l'ostentation. Or elle apparaît pour les grands personnages comme un moyen d'affirmer leur suprématie sociale. Pour faire étalage de leur richesse et de leur puissance, ils se doivent donc de tenir table ouverte.

Pour cela ils disposent des capacités de réception nécessaires. Durant des siècles, les festins se déroulaient sur des tables mobiles soutenues par des tréteaux" . Cette habitude perdure chez les élites bordelaiscs, mais ces dernières font aussi preuve de modernité et de souci de confort en adoptant dans la deuxième moitié du XVIII ${ }^{c}$ siècle les salles à manger. Ces pièces réservées auX repas sont présentes dans les hôtels particuliers, comme celui du maître des requêtes Bacalan rue du Mirail ${ }^{32}$. À travers la mention de ces pièces, on peut voir que les élites bordelaises ont la possibilité d'accueillir de nombreux convives.

Ces capacités de réception, plutôt importantes, peuvent aussi être évaluées grâce à la domesticité liée à la table et à la cuisine. Pour cela, les rôles de capitation nous fournissent de précieux renseignements. Ainsi, en 1768, la majorité des membres du parlement de Bordeaux ont au moins une cuisinière à leur service ${ }^{3.3}$. Le président Leberthon quant à lui est entouré de huit domestiques dont un maître d'hôtel, un cuisinier et un marmiton. La présence d'une domesticité aussi abondante est nécessaire à la tenue d'une table de qualité, mais elle témoigne aussi d'une volonté d'affirmer son rang social. Elle $s$ 'accompagne parfois d'un recours à des professionnels de la table comme les traiteurs, qui assurent qualitć gastronomique et prestige social.

L'abondance de la vaisselle souligne elle aussi cette volonté des élites bordelaises d'étaler leur richesse en tenant table ouverte. Dans les inventaires après décès que nous avons dépouillés, le nombre moyen d'assiettes est proche de 118 et celui des serviettes de 255 unités, ce qui laisse supposer l'organisation de repas réunissant de nombreux convives. Dans certains cas, les chiffres peuvent être beaucoup plus élevés, comme chez le maître des requêtes Bacalan où l'on ne trouve pas moins de 1248 serviettes accompagnées de 104 nappes ${ }^{34}$. Cette abondance, synonyme de luxe, se manifeste aussi dans les cuisines. La batterie de cuisine de Madame de Paty par exemple comprend vingt-neuf casseroles, vingt-quatre marmites et six tourtières ${ }^{35}$. Elle n’a donc rien à envier,

30. BALI.VY (O.), art. cit.,p. 125.

31. Pardoll hé-Galabrin (Annick), La naissance de lintime. 3 oon foyers parisiens. XVIT"XVIII siecles, Paris, 1988 , p. 304.

32. ADG. 3 E 15022. 25 décémbre 1772.

33. ADG. C 2729, rôle de capitation des domestiques des membres du parlement de Bordeaux pour l'année 1768 .

34. ADG, 3 E 15022. 25 décembre 1772.

35. ADG, 3 E 5578. 11 avril 1775. 
à titre de comparaison, à celle du seigneur de La Grange, conseiller à la cour des aides de Paris en 1775, où sont inventoriées vingt-cinq casseroles, sept marmites et quatre tourtières ${ }^{35}$. Cette vaisselle en nombre est indissociable de la mise en œuvre du service à la française, symbole d'une recherche permanente de l'abondance. Il permet en effet de présenter sur la table de très nombreux plats afin que chacun puisse choisir ce qui lui convient le mieux ${ }^{37}$. Cette pratique, attestée par Madame de La Roche ${ }^{38}$, est confirmée par les menus où sont mentionnés souvent une vingtaine de plats différents.

Toutefois cette fonction de représentation de la table ne saurait se résumer à un amoncellement de vaisselle et d'ustensiles de cuisine. Elle doit aussi s'exprimer par une recherche de la rareté et de la qualité parmi les objets de table et les mets consommés. Les élites bordelaises apprécient tout particulièrement les matières précieuses telles que l'argenterie, comme en témoignent les nombreuses pièces conservées dans les musés de la ville ${ }^{39}$. Arthur Young souligne que «de grands dîners [y sont] servis dans de la vaisselle plate ${ }^{+0}$ », c'est-à-dire en argent. Il n'est donc pas surprenant de la retrouver très présente dans les inventaires après décès. Ainsi, chez le conseiller au parlement Étienne de Gombault, le notaire, très attentif à ces objets de valeur, décrit la vaisselle en argent qui comprend entre autres un service de quarante-huit assiettes accompagné de vingt-quatre fourchettes, cuillères et couteaux ${ }^{41}$. De manière générale, même si le nombre d'objets en argent est moins important, on trouve dans la plupart des cas au moins une salière, un sucrier ou une écuelle en argent, signe d'une volonté marquée des élites d'afficher le luxe de leur table.

La consommation de produits onéreux est aussi le signe d'une véritable hiérarchisation sociale des mets ${ }^{42}$. Une lettre adressée au président de Lalanne ${ }^{43}$ indique que la livre de truffes coûte trois livres, ce qui en fait un produit plutôt cher, réservé à la table des élites. Or, ces truffes sont très présentes dans les menus de la jurade ou de la chambre de commerce, servies seules ou comme accompagnement de volailles telles que chapons ou poules-dindes. Ce goût pour les truffes ne provient donc pas uniquement, comme nous avons pu le voir, des potentialités offertes par le milieu naturel et l'arrière-pays agricole ; il répond aussi à la volonté de se distinguer socialement par la consommation de produits coûteux.

36. Pardail.hé-Galabrun (Annick), op. cit., p. 290.

37. Mabil.t.e (G.), « 1690-1800. La table à la française », dans Histoire de la table, Paris, 1994.

38. Meaudre de LAPOUYADF, art. cit., p. 269 : " chacun prit et offrit de ce qu'il voulut de chacun des plats qui étaient devant lui $»$.

39. Coustet (R.), Bordeatix, l'art et le vin, Bordeaux, 1995, p. 107.

40. Young (Arthur), Voyages en France én 1787, 1788, 1789, publiés par H. Sée, Paris, 1931, p. 139.

41. ADG, 3 E 6803, 23 juillet 1721.

42. Marenco (Cl.), Manières de table, modeles de móurs (XVII'XX' siécles), Cachan, 1992.

43. "Lettre adressée au président de Lalanne à l'occasion de l'envoi d’une poule-dinde et de truffes le 2 juin 1759 ». Archives historiques de la Gironde, t. 45, p. 458. 
Dans cette perspective, la rareté ou l'originalité des aliments sont des signes sociaux forts. La consommation de fruits hors saison est une forme de luxe particulièrement appréciée par les élites bordelaises du XVIII ${ }^{c}$ siècle. Comme en témoigne son livre de comptes, François-Léon de Galatheau n'hésite pas à manger des compotes de poires en février ou des raisins de Calabre en mars ${ }^{44}$. Le goût du luxe devient alors une source d'identité culinaire pour la table bordelaise. Cependant la distinction la plus nette ne se fait pas dans cet étalage de richesse, mais plutôt dans un raffinement synonyme de bon goût.

\section{Le bon gô̂t des élites}

Le bon goût est une notion caractéristique de la cuisine du XVIII ${ }^{c}$ siècle ${ }^{+5}$. À travers le raffinement de leur table, les élites cherchent à se distinguer du reste de la société, à l'alimentation plus monotone et aux mets plus grossiers. Elles participent ainsi à la mise en place d'une gastronomie locale originale.

La spécialisation et la rareté des objets de table peuvent être un signe de raffinement. Un certain nombre d'inventaires après décès nous en ont offert des exemples intéressants. Le bon goût peut s'exprimer par la personnalisation des couverts, comme ces " couteaux à manche d'argent aux armes du seigneur ${ }^{+6} \gg$. La présence de tire-moelle ou de marteau à sucre chez l'ingénieur du roi Saint-André ${ }^{47}$ confère à sa table une image de bon goût liée à la sophistication de ces ustensiles. Le raffinement peut aussi apparaître de manière symbolique à travers un goût pour des couleurs particulières, comme le bleu ou le rouge. Qu'il s'agisse de service en grès bleu, comme sur la table du négociant Dargenton ${ }^{+8}$, ou de serviettes à café de Béarn rouges et bleues, utilisées par de Laroque seigneur de Latour ${ }^{+9}$, nombreuses sont les références à ces couleurs, symboles de noblesse et de richesse ${ }^{50}$. Ce goût de l'ostentation à travers les arts de la table répond bien à la recherche du luxe des grandes familles de Bordeaux évoquée par François de La Rochefoucauld ${ }^{51}$.

Les choix alimentaires et les préparations préférées des élites bordelaises correspondent aussi aux règles du bon goût. On peut observer une préférence pour les viandes, et le gibier en particulier. Ainsi sont régulièrement servis des perdreaux, des levrauts, des bécassines sous forme de rôts, mais aussi dans des

44. AMB, fonds Beaumartin. dossier XLII. F.-L. de Galatheau, livre de comptes.

45. FLANDRIN (Jean-Louis), « De la diététique à la gastronomie, ou la libération de la gourmandise ", dans Flandrin (Jean-Louis) et Montaniari (Massimo) dir.. Histoire de l'alimentation. Paris, 1996, p. 683-703.

46. ADG, 3 E 24879, 12 juillet 1773.

47. ADG, 3 E 13064,11 janvier 1775.

48. ADG, 3 E 6230, 20 août 1770.

49. ADG, 3 E 5566, 11 novembre 1771.

50. Rowı.t.y (Anthony), Les Français à table. Atlas historique de la gastronomie française. Paris, 1997,p. 16.

51. Voyages en France de François de la Rochefoucauld. éd. J. Marchand, Paris, 1938, p. 119. 
plats composés comme des terrines d'innocents, des pâtés chauds de bécasses ${ }^{52}$ ou une tourte de pigeons garnie au ris de veau ${ }^{53}$. Nous pourrions multiplier les exemples de plats à base de gibier, mais il en ressort surtout une prédilection pour le gibier à plumes. En effet, ce gibier volant est considéré comme plus raffiné et plus délicat que les animaux vivant au ras du sol, comme les lapins jugés plus vulgaires et rarement consommés. Le repas servi à la chambre de commerce le 15 septembre $1753^{5+}$ illustre bien le goût des notables en matière de gibier, puisqu'on y déguste une entrée de bécasses, trois perdreaux et dix bécassines. Ces menus de saison de chasse sont vraiment une bonne illustration de la conception nobiliaire de la table partagée par les élites bordelaises. Lc banquet donné par la chambre de commerce en septembre $1775^{55}$ confirme la suprématie de la viande (seize plats sur vingt-cinq), mais surtout le goût pour un gibier de saison (lièvres, bécasses, perdreaux), synonyme de prestige social. Les préférences alimentaires ne sont donc pas uniquement guidées par des critères culinaires, mais répondent aussi souvent à un besoin de distinction sociale.

Les modes de préparation les plus appréciés s'inscrivent dans le même processus. Faisant souvent appel à des professionnels de la cuisine, comme nous l'avons vu plus haut, les élites bordelaises recherchent les préparations complexes comme les pâtisseries, au sens ancien du terme qui désigne tous les plats à base de pâte. Nous pouvons ainsi découvrir des "tourtes de pigeons au ris de veau" ${ }^{56}$ ", des "vol au vent d'agneau garnis ${ }^{57}$ ", ou des terrines de lapereaux ${ }^{5 x}$. Ces terrines à base de gibier tiennent souvent une place importante dans les menus. Ce goût pour les pâtés, terrines et autres tourtes est confirmé par la présence de tourtières dans $80 \%$ des inventaires après décès. La recherche du bon goût s'exprime donc dans la réalisation de plats complexes, mais on peut aussi la retrouver dans la dénomination même des plats proposés. Parmi les chefs-d'œuvre des maîtres hôteliers ${ }^{59}$, on peut rencontrer des « fricassées de poulet à la Pompadour » ou des plats «d'oreilles de veau à la poivrade », illustration du raffinement gastronomique bordelais.

Gibiers à plumes et pâtisseries sont autant d'aliments qui ont une forte connotation sociale. Les élites bordelaises cherchent ainsi à se conformer à leur rang et à affirmer leur suprématie. Cependant, au delà de cette volonté commune de se différencier par la table, elles développent des habitudes alimentaires variées afin de se distinguer entre elles.

52. AMB , CC 1114 .

53. ADG, C 1764

54. ADG, C 4421

55. ADG. C 4424.

56. АDG. C 1764, délibération du 2 juin 1760.

57. Ibid. 7 février 1786 .

58. ADG. C 4421, repas du 6 septembre 1753.

59. ADG. C 1764 
Comme enjeu des rapports sociaux, la table trouve dans le Bordeaux du XVIII' siècle un terrain plutôt propice. En effet, la présence d'une noblesse parlementaire puissante et d'un monde du négoce en plein essor offre un cadre favorable au développement d'une gastronomie locale, fruit de la concurrence mais aussi du mimétisme social entre ces deux groupes. L'alimentation est manifestement un outil approprié à la compétition entre la fraction dominante et la fraction ascendante de la sociétét(t). L'évolution de la cuisine bordelaise du XVIII' siècle est fortement influencée par ce phénomène.

Si l'on devait établir une hiérarchie des meilleures tables de la ville à cette époque, celles de la noblesse parlementaire se placeraient sans aucun doute en tête. En effet, si on se réfère aux inventaires après décès qui constituent la source la mieux adaptée pour saisir les élites dans leur diversité, on constate la présence récurrente chez ces parlementaires d'éléments permettant de tenir table ouverte dans le luxe et le raffinement. L'inventaire après décès du maître des requêtes Bacalan' ${ }^{61}$ par exemple comprend 320 assiettes, 36 couverts, 67 plats, 13 casseroles et 1248 serviettes, qui lui donnent la possibilité de recevoir aisément de nombreux convives. Comme nous l'avons déjà vu, les parlementaires sont aussi entourés de nombreux domestiques, symboles de leur supériorité sociale.

D’autre part, cette noblesse se place au cœur des innovations culinaires de son époque. Dès les années 1720-1725, on trouve des assiettes creuses chez des conseillers au parlement comme Fayard ${ }^{62}$ ou Gombault ${ }^{6.3}$, alors qu'elles sont ignorées de la plus grande partie de la société. Ces parlementaires font aussi preuve d'un goût raffiné en matière de vin. La cave du maître des requêtes Bacalan compte, entre autres, des tonneaux de vin rouge de Pessac ${ }^{6+}$ dont la renommée est déjà établie au XVIII ${ }^{c}$ siècle avec, par exemple, le cru du château Haut-Brion, propriété de grands parlementaires ${ }^{65}$.

La table de la noblesse parlementaire bordelaise se distingue donc par un luxe et un raffinement supérieurs à ceux du reste de la société. Dans une ville où « le devoir de munificence est une nécessité aristocratique ${ }^{60}$ », elle est donc bien un modèle pour le reste des élites.

6). Fischi_z (Cl.), L'Hommivore, Paris, 1990 , p. 176.

61. ADG. 3 E 15022. 25 décembre 1772.

62. $\triangle \mathrm{DG}, 3$ E 7991,4 mai 1720 .

63. ADG, 3 E 6803.23 juillet 1721 .

64. ADG, 3 E 15022. 25 décembre 1772.

65. Butri. (Paul), "Grands proprićtaires et production des vins du Médoc au XVII" siècle ". Relue historique de Bordeaux. 1963.

66. GIRARI) (A.), «Le triomphe de "la cuisinière bourgeoise". Livres culinaires. cuisine el société en France aux XVII" et XVIIJ" siecles". Revue dhistoire moderne ét contemporaine. octobre-décembre 1977. p. 513. 
Pour les négociants, qui constituent le deuxième groupe important de ces dernières, la table est un moyen de se rapprocher de l'idéal nobiliaire. Mimétisme et compétition sociale participent alors à l'émergence de goûts alimentaires et de manières de table originaux.

Leur table se caractérise tout d'abord par un goût moins marqué de l'ostentation et de l'opulence, qui apparaît bien dans les inventaires après décès où la vaisselle et les ustensiles de cuisine sont moins nombreux et moins diversifiés que chez les nobles. Les inventaires des élites du commerce ne comprennent en moyenne que 104 assiettes, alors que ceux des nobles atteignent 130. Le nombre de plats s'inscrit dans la même logique puisque le nombre moyen est de vingt-huit pour le négoce contre quarante pour la noblesse. Ces indications permettent donc de penser que le monde du commerce a une sociabilité à table plus réduite et qu'il est plus attiré par des repas en petit groupe au luxe moins affirmé. Les élites négociantes conservent aussi des manières de table délaissées par les modes nouvelles. Les mazarines, par exemple, sont un modèle d'assiettes à bassin très profond et larges bords qui tend à disparaître au cours du XVIII siècle $^{67}$. On les retrouve pourtant dans $12,5 \%$ des inventaires des négociants dans les années 1770 , alors qu'elles ont complètement disparu des inventaires nobiliaires depuis 1720-1725. La même tendance se retrouve avec les vinaigriers, objets révélateurs eux aussi de goûts culinaires dépassés. Le maintien de l'utilisation de ces objets témoigne sans doute d'une volonté des élites marchandes d'adopter les goûts de la noblesse, mais avec un certain décalage chronologique.

Toutefois, cela n'empêche pas le milieu négociant d'être à la pointe de certaines évolutions culinaires. Il joue, par exemple, un rôle précurseur dans l'adoption du café, qui va par la suite imprégner l'ensemble de la société. Ainsi, dès les années 1720-1725, les cafetières sont mentionnées dans la moitié des inventaires après décès des négociants, alors que chez les nobles elles n'apparaissent que dans $37,5 \%$ des cas. À la même époque, les poêles à café utilisées par les marchands sont totalement absentes des cuisines nobiliaires. Sensibles aux modes culinaires venues de Hollande, d'Angleterre ou d'Allemagne, le milieu négociant bordelais, souvent d'origine étrangère, manifeste ainsi un goût précoce pour le café et participe à sa diffusion parmi les élites de la ville.

Pour les négociants, la table peut donc être un moyen de rivaliser avec les élites nobiliaires. Les repas organisés à la chambre de commerce, symbole de la puissance du négoce, illustrent assez bien ce phénomène. La venue de personnages importants, comme l'intendant ou le gouverneur général de la Martinique, est l'occasion de grands festins préparés par des traiteurs ${ }^{68}$. Le dîner servi le 15 septembre $1775^{(0)}$ est très représentatif de la volonté du négoce

67. GIRARD (S.), Histoire des objets de cuisine et de gourmandise, Paris, 1991.

68. ADG, C 4424.

69. Idem. 
d'imiter le comportement alimentaire de la noblesse. Lors de ce repas, qui ne comporte pas moins de vingt-six plats différents, sont proposés aux convives du gibier, avec des lapereaux et des perdrix, des plats fastueux comme un chapon farci aux truffes, et un grand nombre de plats à base de viande. Tout ceci concourt à montrer que la table des négociants n'a rien à envier à celle de la noblesse et que cette compétition sociale permanente participe à l'affirmation de l'identité gastronomique bordelaise.

Cependant le luxe et le raffinement de la table des parlementaires et des riches négociants ne doivent pas faire oublier la profonde diversité des pratiques alimentaires à l'intérieur de groupes sociaux en apparence homogènes. Le milieu nobiliaire connaît ainsi des habitudes culinaires très différentes.

Malgré ses titres d'écuyer et de seigneur de Pelleau, la table de Jean-Joseph de Pelleau ${ }^{70}$ est bien loin du luxe et de l'opulence de celle des parlementaires. Sa modeste argenterie se compose de douze cuillères et fourchettes, d'une écuelle, d'une cuillère à potage et de deux salières. Sa vaisselle est essentiellement en étain et sa petite douzaine de plats peuvent laisser supposer que ce petit noble d'épée ne peut guère organiser des festins gastronomiques. La diversité des pratiques alimentaires rejoint donc bien la diversité sociale, même au sein des élites.

Malgré une volonté commune de se distinguer du reste de la société par des goûts culinaires raffinés et un luxe affiché de la table, les élites bordelaises sont loin de former un groupe homogène. La table d'un grand parlementaire, recouverte de gibiers, de pâtisseries et de truffes, servie par une foule de domestiques, n'a rien de commun avec l'alimentation plutôt monotone d'un petit noble. La table est bien l'enjeu d'une compétition sociale entre une élite nobiliaire attachée à préserver sa suprématie par le luxe et le bon goût, et un monde du négoce avide d'idéal nobiliaire mais désireux d'affirmer ses spécificités culturelles. De leur confrontation naît une évolution contrastée des habitudes alimentaires.

\section{Entre tradition et modernité}

Ces termes recouvrent deux tendances majeures qui caractérisent bien la table bordelaise. Afin de bien percevoir sa spécificité, il convient de la comparer à l'évolution générale des pratiques alimentaires dans un XVIII" siècle marqué par l'émergence d'une «nouvelle cuisine »" ${ }^{71}$ Pour cela, nous avons choisi, à travers nos inventaires après décès, de nous intéresser à deux périodes charnières. Nous avons donc réalisé une comparaison entre les

70. ADG, 3 E 24848, 16 juin 1724.

71. Whiton (B. K.), L'office et la bouche. Histoire des ma'urs en France, 1300-1789. Paris. 1984. p. 243. 
actes des années 1720-1725, lendemain de la Régence et date d'affirmation de la gastronomie, et ceux des années 1770-1775, apogée du dynamisme bordelais mais aussi des principes de la «nouvelle cuisine». Face à des changements majeurs, tels que l'émergence de saveurs plus douces ou de pratiques plus individuelles, les élites bordelaises ont des attitudes variables, oscillant entre un rôle novateur, dans leur goût pour le vin vieux par exemple, et un certain archaïsme, avec le maintien de saveurs anciennes et un goût modéré des légumes.

\section{Une table de son temps}

Afin de conserver une fonction de distinction sociale à travers leurs habitudes alimentaires, les élites se doivent de suivre, voire d'impulser, les innovations dans le domaine de la table. Modèles sociaux, elles participent pleinement à la construction et à l'affirmation d'une gastronomie locale originale.

L'évolution des manières de table au XVIII" siècle se caractérise par un souci nouveau de l'hygiène et un individualisme accru des pratiques. Le service de table et l'évolution de la vaisselle sont deux phénomènes qui démontrent que les élites bordelaises sont bien au cœur de ces changements.

L’étude du nombre de domestiques, tel qu“il apparaît dans les rôles de capitation, souligne un goût nouveau pour des repas plus intimes où la domesticité se fait plus discrète, laissant place à des manières de table moins solennelles. Ainsi, en 1702, le premier président du parlement a pour son service quinze domestiques dont un sommelier, un maître d'hôtel, un cuisinier et un garçon de cuisine ${ }^{72}$. Le président Leberthon, en 1768, n'a plus qu'un maître d'hôtel, un cuisinier et un marmiton parmi seulement huit domestiques ${ }^{73}$. La disparition de la fonction de sommelier souligne l'allégement du service.

Toutefois, c'est à travers le service des boissons que l'on voit sans doute le mieux apparaître ce phénomène. En effet Franklin, dans le volume de La vie privée d'autrefois consacré au repas, signale que les verres et les bouteilles restent sur les tables à partir de $1760^{74}$. Par conséquent, cette nouvelle habitude restreint le rôle des serviteurs, qui jusqu'alors devaient remplir le verre de chaque convive durant le repas avec des bouteilles placées en dehors de la $\operatorname{table}^{75}$. À Bordeaux, cette nouvelle manière de servir est attestée par la multiplication des verres tout au long du siècle. En effet, alors qu'en 1720-1725 les verres ne sont mentionnés que dans $18,75 \%$ des inventaires, en 1770-1775 ce

74. Franki.N (A.), La vie privée d'autrefois : les repas, Paris, 1889.

75. Maвm.l.: (G.). "Le service de la boisson à table ", dans $X V I I J$ siécle, numéro spécial sur le vin. 1997, p. 147. 
taux atteint 68,75\%. L'essor des seaux à verres et à bouteilles montre bien aussi que ceux-ci sont laissés de plus en plus souvent sur les tables, ce qui implique une intervention moins fréquente des domestiques et donc une plus grande intimité pour les convives. Dans ce domaine, les Bordelais suivent les évolutions du siècle. Cependant, cette attitude nouvelle ne peut se réaliser sans une individualisation de plus en plus grande des manières de table.

Ce souci de l'hygiène à table est une innovation, puisque jusqu'au XVIII ${ }^{\circ}$ siècle ce problème a été le plus souvent ignoré. Le lien entre recherche de l’intimité et souci d'hygiène se manifeste très bien dans le service du potage. En effet, jusqu'au XVIII' siècle, chacun se servait avec sa propre cuillère dans un même plat. Or on constate dans les inventaires après décès le développement d'une vaisselle permettant de manger des potages de manière plus individuelle. On remarque tout d'abord que les assiettes creuses, apparues à la fin du XVII siècle $^{76}$, sont déjà présentes dans les inventaires bordelais des années 1720-1725. La diffusion des cuillères à potage confirme cette préoccupation nouvelle. En effet, la diffusion sur les tables de ces nouveaux objets permet aux convives de se servir, puis de manger dans leurs propres assiettes creuses sans être obligés de manger dans le plat commun.

L'engagement de la table bordelaise dans l'évolution des nouvelles manières de table est enfin confirmée par la croissance des couverts spécialisés. Le terme même de couvert, inconnu dans les années 1720-1725, est présent dans $43.75 \%$ des inventaires de la période 1770-1775. Les couverts spécialisés, comme les couteaux à dessert ou les cuillères à sucre, tendent à se multiplier tout au long du siècle. Ces deux couverts, inconnus dans les années 1720-1725, sont mentionnés respectivement dans $18,75 \%$ et $31,35 \%$ des actes de la période 1770-1775. Cette diffusion d'objets qui évitent le partage des couverts entre les divers convives montre bien que les élites bordelaises sont attentives aux changements de leur époque, condition nécessaire au maintien de leur suprématie sociale. L'art culinaire bordelais suit lui aussi le renouveau de son temps.

En effet, loin d'être immuable, la cuisine connaît des évolutions permanentes et le XVIII' siècle constitue un tournant dans ce domaine, avec la diffusion des boissons exotiques, la place croissante des légumes verts ou encore l'abandon progressif des épices. Nous allons donc chercher à savoir, à travers quelques exemples, comment la table des élites bordelaises s'inscrit dans ce processus.

Elles manifestent un goût croissant pour les viandes de boucherie. Le livre de comptes de François-Léon de Galatheau confirme l'achat régulier de mouton, de veau et de bœuf dans les années $1770^{77}$. De même, les repas servis à la chambre de commerce comportent régulièrement de la viande de boucherie, sous forme de filets de bœuf, de carrés de veau glacés ou de côtelettes

76. GIRARI) (S.).op. (it

77. AMB, fonds Beaumartin, dossier XLII, F.-L, de Galatheau, livre de comptes. 
d'agneau aux fines herbes ${ }^{7 \$}$. Or ces viandes, jugées grossières par la diététique, ont longtemps été abandonnées aux classes populaires. Dans ce domaine, la cuisine du XVIII' siècle marque une rupture en intégrant ces viandes aux menus des élites, mais en utilisant seulement les meilleurs morceaux. Ainsi, parmi les chefs-d'œuvre des maîtres hôteliers de Bordeaux, nous pouvons rencontrer des plats réalisés à partir des morceaux les plus fins, comme une épaule de mouton aux pistaches ${ }^{79}$, un gigot à la royale ${ }^{80}$ ou une noix de veau garnie de ris de veau ${ }^{81}$. Ce goût nouveau pour les viandes de boucherie s'accompagne d'un retour des herbes indigènes destinées à pallier la disparition progressive des ćpices exotiques.

Longtemps prédominantes dans la cuisine européenne, en particulier à l'époque médiévale ${ }^{82}$, les épices comme le safran connaissent un net recul dans la cuisine des élites du XVIII" siècle. Cette évolution suivie à Bordeaux apparaît clairement parmi les chefs-d'œuvre des maîtres hôteliers ${ }^{83}$. On peut trouver, dans la seconde moitié du siècle, un fricandeau à l'oseille, une longe de veau à l'estragon, des pigeons au basilic ou une omelette au persil. Le menu de la chambre de commerce daté du 15 septembre 1775 comprend un plat de pieds de mouton au basilic ${ }^{8-}$. Cette modernisation des pratiques culinaires correspond à la volonté nouvelle de préserver la saveur propre des aliments. On constate par exemple que les sauces sont servies de plus en plus souvent à part dans des sauciers ; inconnus dans les années 1720-1725, ils apparaissent dans $56,25 \%$ des inventaires après décès en 1770-1775.

Goût pour les herbes indigènes, volonté de préserver les saveurs sont autant d'indications qui confirment que la table des élites bordelaises suit les évolutions du siècle. Nous allons maintenant voir que, dans le domaine des boissons, elles peuvent même être à la pointe de la mode.

\section{Une table à la pointe de la mode}

Boissons exotiques et vins de qualité ont en effet pendant longtemps fait la réputation de Bordeaux. Port de commerce et capitale d'un grand vignoble, la ville a tous les atouts pour être un précurseur dans l'adoption de ces nouvelles habitudes alimentaires.

En effet, comme nous avons pu l'observer à propos des élites négociantes, le café fait une apparition précoce mais surtout très marquée au XVIII ${ }^{\mathrm{e}}$ siècle. Ce

78. ADG, C 4421 .

79. ADG, C 1764. délibération du 18 février 1772.

80. Ibid., 9 février 1773.

81 . Ibid., 17 juin 1789.

82. Laurioux (B.), Le règne de Taillevent. Livres et pratiques culinaires à la fin du Moven Âge, Paris, 1997. 
phénomène est attesté dès le début du siècle par une ordonnance de police datée du 29 mai 1708 stipulant que les cafés doivent être fermés à neuf heures du soir en hiver et dix heures en été ${ }^{85}$. Les poêles à café , mentionnées dans $25 \%$ des inventaires de la période 1720-1725, atteignent le chiffre de 68,75\% dans les années 1770-1775. À la même époque, les cafetières sont présentes dans $93,75 \%$ des cas. Tous ces éléments confirment le goût particulier des élites bordelaises pour ce nouveau breuvage.

Cependant, c'est sans doute à travers une préférence pour le thé qu'elles affirment le mieux leur distinction sociale, leur rôle novateur et donc leur identité culinaire. Si on se réfère aux inventaires après décès que nous avons dépouillés, on observe que, mentionnées dans 12,5\% des actes des années 1720-1725, les théières se retrouvent dans $87,5 \%$ des inventaires de la période 1770-1775. Cette tendance pourrait aussi être évaluée par la multiplication des boîtes à thé dans les offices des hôtels particuliers. Or, il est intéressant de souligner que la consommation de thé en France au XVIII ${ }^{c}$ siècle est surtout l'apanage des élites parisiennes, en particulier durant les années 1720-1730 ${ }^{86}$. Son usage semble demeurer très rare en province ${ }^{87}$. Les élites bordelaises sont donc bien à la pointe de l'innovation culinaire en ayant un goût pour le thé dès le début du siècle, mais surtout elles se distinguent par le maintien de sa consommation. Le goût pour le thé est donc un trait distinctif de la table locale.

Toutefois, comment parler du rôle précurseur des élites bordelaises du XVIII ${ }^{\circ}$ siècle au niveau des boissons sans nous attarder sur la plus prestigieuse d'entre elles, le vin ? Afin de bien saisir comment les élites de la ville ont été novatrices dans ce domaine, nous pouvons étudier le développement du goût pour des vins de qualité bonifiés par les années. Celui-ci se réalise, dans un premier temps, par de nouvelles conditions de conscrvation. Les inventaircs après décès soulignent, par exemple, une diffusion précoce et rapide des bouteilles en verre. Dans son livre de comptes, à la date du 23 mars 1729, Raymond de Navarre note l'achat de cinq douzaines de bouteilles en verre destinées à la conservation du vin ${ }^{88}$. Pour Gilbert Garrier, l'utilisation de la bouteille ne se généralise que dans la seconde moitié du XVIII ${ }^{\mathrm{e}}$ siècle $^{89}$. Les élites bordelaises engendrent donc des modes nouvelles.

Cette conservation du vin en bouteille offre des possibilités nouvelles de vieillissement. Dès 1730 , selon son livre de raison, Raymond de Navarre procède à l'achat de deux tierçons de vin de Saint-Émilion de la récolte de $1722^{90}$. Le goût des élites bordelaises pour les vins vieux est souligné à la

85. AMB, FF 72, police des hôtels, cafés, cabarets.

86. PARDAIL.hÉ-Gal ABRUN (Annick), op. cit. , p. 301.

87. Hultz de LEMPS (A.), «Boissons coloniales et essor du sucre », dans Histoire de l'alimentation, op. cit., p. 639.

88. AMB, fonds Beaumartin, dossier XII (716).

89. GARRILR (Gilbert), Histoire sociale et culturelle du vin, Paris, 1995, p. 134.

90. AMB, Fonds Beaumartin, dossier XII, livre de raison, 6 mars 1730. 
veille de la Révolution par Thomas Jefferson, qui remarque que les vins blancs bordelais peuvent être conservés quinze à vingt ans"'. L'inventaire de la cave de l'ingénieur du roi Saint-André en $1775^{\circ 2}$ fournit une illustration intéressante de ce goût nouveau. En effet, elle contient tout d'abord un nombre impressionnant de bouteilles en verre, indispensables au vieillissement. Ensuite, le notaire mentionne explicitement la présence de « 110 bouteilles de Saint-Émilion vieux », mais aussi celle de neuf tierçons de vin rouge du même cru de l'année 1773, ce qui témoigne de l'intérêt nouveau porté au millésime des vins. Il est intéressant de noter ici que les vins rouges semblent être les plus apprécié, au regard des quantités conservées. À travers l'exemple de la cave de cet officier royal séjournant dans la ville pendant quelques années, on constate que les goûts novateurs des Bordelais en matière de vin sont repris le plus souvent par l'ensemble des élites.

Comme le prouve le goût partagé des boissons exotiques et des vins de qualité, les élites de Bordeaux, dans leurs choix alimentaires, oscillent entre ouverture vers l'extérieur et attachement au terroir. La table bordelaise est ainsi faite de contradictions qui construisent son identité. Il n'est donc pas surprenant de constater que, malgré certains aspects novateurs, les habitudes culinaires des élites bordelaises demeurent marquées par les saveurs anciennes.

\section{Le poids de la tradition}

Certaines modes alimentaires, parfois délaissées ailleurs et le plus souvent rejetées par la «nouvelle cuisine », sont des traits distinctifs de la gastronomie bordelaise. Ce poids de la tradition apparaît en cette fin de XVIII" siècle comme le reflet d'une société attachée à ses prérogatives et soucieuse de maintenir son prestige social et son identité.

La pérennité d'un goût pour la viande, aliment à forte connotation sociale, $s$ 'inscrit pleinement dans cette perspective. La présence dans les inventaires après décès des années 1770 d'objets tels que des balances ${ }^{9.3}$ ou des crochets à viande ${ }^{94}$, et de manière plus générale des lèchefrites, confirme la place toujours importante de la viande dans l'alimentation des élites bordelaises, alors que cette pratique culinaire semble aller à l'encontre de l'évolution générale. Elles se singularisent par rapport aux modes parisiennes et aux principes de la «nouvelle cuisine » par un goût prononcé pour le porc sous toutes ses formes. En effet, alors que sa consommation est en net recul dans les hautes classes de la société française ${ }^{95}$, le porc, et les abats en particulier, demeurent très prisés à

91. Ginestet (B.). op. cit. , p. 40.

92. ADG. 3 E 13064. 11 janvier 1775.

93. ADG. 3 E 5578. 11 avril 1775.

94. ADG. 3 E 13064.11 janvier 1775.

95. Fi.A.VIBRIN (Jean-Louis). op. cit.. p. 277. 
Bordeaux même à la fin du XVIII ${ }^{*}$ siècle. Ainsi, parmi les plats servis à la chambre de commerce ${ }^{96}$, on rencontre souvent des saucisses, des andouilles ou des boudins. Les pieds de pore «à la Saint-Mehou» ou à la dauphine et les plats de langues apparaissent aussi de manière régulière. L'omniprésence de la viande sur la table des élites bordelaises révèle une volonté de maintenir leur prestige social, mais aussi le poids des traditions culinaires.

Cette part importante de la viande dans l'alimentation ne saurait être concurrencée par les nouveaux produits et les nouvelles saveurs en vogue ailleurs. Les notables bordelais manifestent par exemple une certaine réticence vis-à-vis des légumes, pourtant mis à l'honneur dans la «nouvelle cuisine » de l'époque. Les légumes verts sont quasiment absents des sources et n'apparaissent dans les chefs-d'œuvre des maîtres hôteliers" ${ }^{17}$ qu'à la fin du siècle, sous forme de plats d'épinards «à la belle veuve " ou au naturel. Seuls les féculents tiennent une place importante, puisque les ragoûts ou les potages de pois sont régulièrement présentés sur les tables.

De même, le goût modéré des élites bordelaises pour les desserts ne saurait mettre à mal leur prédilection pour les viandes. Dans les années 1760-1770, les chefs-d'œuvre des maîtres hôteliers ne sont jamais des plats de dessert; il faut attendre les années 1780 pour voir se multiplier des préparations telles que des charlottes, des tourtes à la frangipane aux amandes ou des crèmes soufflées. Cette discrétion des desserts, la place réduite des légumes et le maintien d'un goût prononcé pour la viande, pour le porc en particulier, sont autant de tendances qui vont à l'encontre de l'évolution d'ensemble de la cuisine du XVIII ${ }^{\circ}$ siècle. Le menu du 15 septembre $1775^{\circ 8}$ résume bien le poids des traditions dans la cuisine bordelaise. La viande est présente dans plus de la moitié des plats; on y relève la mention de pieds de cochon toujours appréciés par les élites. Les légumes sont plutôt rares : seuls des choux-fleurs et des cardes sont servis. Quant au dessert, le peu de détail fourni peut laisser penser qu'il devait tenir une place minime dans le repas. Ce menu est donc bien le reflet de l'identité culinaire bordelaise.

Celle-ci s'exprime aussi par un goût des élites pour certaines saveurs anciennes. Rejetées au profit des sauces grasses et de la douceur des mets sucrés par la «nouvelle cuisine», les saveurs acides par exemple demeurent très appréciées par les grands personnages bordelais. Dans les menus de la chambre de commerce et de la jurade, les huîtres vertes sont mentionnées très régulièrement. Ces dernières, servies crues et avec du poivre selon la recette de Menon $^{\text {'9 }}$, sont sans doute représentatives d'un goût de l'acidité. Certaines recettes conservées parmi des papiers de famille, comme celle des noix au 
vinaigre ${ }^{1(x)}$, confirment ce phénomène. En effet, cette préparation se compose entre autres de gingembre, de clous de girofle et de crème de moutarde mélangés à du vinaigre, ce qui doit donner une certaine note d'acidité, représentative de saveurs anciennes le plus souvent délaissées ailleurs.

Les goûts alimentaires des élites bordelaises du XVIII ${ }^{\mathfrak{e}}$ siècle oscillent donc entre deux tendances contradictoires. Novatrices dans le domaine des boissons exotiques et du vin, elles restent, dans le même temps, attachées à des habitudes anciennes de la cuisine aristocratique comme l'abondance des viandes ou le goût des saveurs acides. Reflet de leur diversité, ces contradictions sont sans doute aussi une source majeure d'originalité pour la cuisine bordelaise.

La table bordelaise du XVIII ${ }^{*}$ siècle exprime pleinement sa richesse gastronomique au cœur de l'âge d'or de la ville. Les goûts alimentaires des élites sont le produit de leur diversité sociale et culturelle. Ils sont le reflet d'une noblesse attachée à préserver sa domination à travers le luxe et d'un monde du négoce en plein essor, attiré par l'idéal de vie nobiliaire, mais désireux de conserver sa culture culinaire. La Révolution française représente une rupture dans le fonctionnement de ce système alimentaire. L'essor progressif de la bourgeoisie et le relatif déclin de la noblesse donneront à la cuisine bordelaise une orientation différente.

Y a t-il donc une identité culinaire bordelaise au XVIII' siècle ? La recherche permanente de l'abondance, l'imprégnation anglo-saxonne de la table avec le goût pour la bière, le thé ou les liqueurs, la présence des produits régionaux comme les huîtres, les truffes ou le gibier à plumes, et bien sûr la consommation de vins de qualité sont indiscutablement des traits distinctifs de la gastronomie bordelaise. Toutefois, la définition de l'identité culinaire locale devrait envisager à la fois l'analyse de cette cuisine raffinée et celle d'une alimentation plus populaire, génératrice elle aussi de spécificités culinaires régionales. Malheureusement, les sources sont souvent muettes sur cette cuisine quotidienne de la majorité de la population bordelaise. 